\title{
Target prostate biopsies: How best to report in synoptic format?
}

Michelle R. Downes, MD'; John R. Srigley, MD²; Andrew Loblaw, MD³ ${ }^{3}$ Nathan Perlis, MD ${ }^{4}$; Sangeet Ghai, $\mathrm{MD}^{5}$; Theodorus van der Kwast, $\mathrm{MD}, \mathrm{PhD}^{6}$

${ }^{1}$ Division of Anatomic Pathology, Laboratory Medicine \& Molecular Diagnostics, Sunnybrook Health Sciences Centre and University of Toronto, Toronto, ON, Canada; ${ }^{2}$ Trillium Health Partners and University of Toronto, Mississauga, ON, Canada; ${ }^{3}$ Department of Radiation Oncology, Sunnybrook Health Sciences Centre, University of Toronto, Toronto, ON, Canada; ${ }^{4}$ Department of Surgery, Division of Urology, University Health Network, Princess Margaret Cancer Centre, University of Toronto, Toronto, ON, Canada; ${ }^{5}$ Joint Department of Medical Imaging, University Health Network, University of Toronto, Toronto, ON, Canada; ${ }^{6}$ Department of Pathology, University Health Network, Toronto, ON, Canada

Cite as: Downes MR, Srigley JR, Loblaw A, et al. Target prostate biopsies: How best to report in synoptic format? Can Urol Assoc J 2021 November 18; Epub ahead of print. http://dx.doi.org/10.5489/cuaj.7460

Published online November 18, 2021

Corresponding author: Dr. Michelle R. Downes, Division of Anatomic Pathology, Laboratory Medicine and Molecular Diagnostics, Sunnybrook Health Sciences Centre, Toronto, ON, Canada; michelle.downes@sunnybrook

$* * *$

\section{Introduction}

The implementation and increasingly widespread use of multiparametric magnetic resonance imaging (mpMRI) in prostate cancer patients is changing clinical practice. Traditional systematic transrectal ultrasound (TRUS) guided biopsy consisting of 10-12 needle cores have been shown to have lower sensitivity than targeted biopsy for detection of clinically significant cancer, ${ }^{1}$ with target biopsies better predicting extraprostatic extension at prostatectomy, ${ }^{2}$ detecting anteriorly located tumors ${ }^{3}$ and identifying perineural invasion. ${ }^{4}$ Further, target biopsy Gleason scores are less likely to be upgraded at prostatectomy compared with TRUS Gleason scores. ${ }^{5}$ The PRECISION trial and the Canadian PRECISE trial, which compared MRI targeted biopsy vs TRUS biopsy in biopsy naïve men, demonstrated the benefit of MRI targeted biopsy in biopsy naïve patients, such that more clinically significant cancers were identified in the MRI targeted arm. ${ }^{2,6}$ The ASIST trial also showed a reduction in upgrading at 2-year follow-up biopsies when patients had baseline MRI targeted biopsies. ${ }^{7}$

MRI targeted biopsies can be either a "stand alone" target biopsy consisting of several cores from a single target, include multiple targets or a combination of target and systematic 
biopsies. Reporting these biopsies in a format that communicates all the relevant information in a concise and accurate manner is crucial. Synoptic reports for prostate biopsies have been in existence in several jurisdictions for many years (International Collaboration Cancer Reporting, Cancer Care Ontario, College of American Pathologists) but the current formats do not account for the evolving change in clinical practice to incorporate prostate target biopsies. The literature to date suggests the optimal method for reporting MRI targeted biopsies is to provide a composite World Health Organization grade (WHO grade)/Gleason score (GS) and cancer extent per target site as opposed to grades/Gleason score for each target core. This method correlates better with both overall tumor volume and extraprostatic extension at prostatectomy. ${ }^{8}$ Additionally, invasive cribriform and intraductal carcinoma (histopathologic features associated with adverse clinical outcomes including biochemical recurrence after prostatectomy and survival), need to be considered in addition to the traditional Gleason score/WHO grade. ${ }^{9}$ While most studies have reported that these adverse features are more likely to be present at MRI sites of disease, ${ }^{10}$ others have concluded that majority of cribriform tumors were non-visible on mpMRI. ${ }^{11}$ Given the contradictory reports, there is uncertainty whether invasive cribriform and intraductal cancer patterns are visible on MRI or not.

The authors (pathologists, urologists, radiologists, radiation oncologists) propose a comprehensive prostate synoptic biopsy report, that can be used for reporting of systematic only, target only or combined target- systematic biopsies (see sample synoptic report below). We have included various data elements based on our experience and practice, which will enable treating clinicians to derive maximal information to drive patient management. The essential elements of such a synoptic report include both a global WHO grade/GS taking into account the grade of all biopsy cores and a composite WHO grade/GS for each separate target component. A variety of additional parameters such as presence/absence of cribriform architecture carcinoma (both intraductal carcinoma and cribriform pattern 4), along with the number of positive sites/cores and total number of sites/cores, perineural invasion, seminal vesicle involvement, periprostatic fat involvement and lymphovascular invasion are also included as per standard synoptic report format.

\section{Synoptic report components}

\section{Histologic type}

Adenocarcinoma (acinar) versus other (e.g. ductal, mucinous). Several subtypes of prostatic adenocarcinoma (eg neuroendocrine, basal/adenoid cystic) do not require a grade/GS hence the need to specify the histologic subtype.

\section{Worst WHO grade/Gleason score}

The literature shows some conflicting results around whether a global or worst grade/GS provides better prognostic information with a global WHO grade/GS showing marginal 
superiority. ${ }^{12,13}$ Some clinicians do utilize the "worst" grade/GS while others prefer the global score. ${ }^{14}$ For this reason, we think inclusion of the worst grade/GS by site or core is of relevance.

\section{Global WHO grade/Gleason score}

We propose inclusion of a global grade to encompass the target and systematic cores, in cases where both are performed. Given the preference for global grade assignment in some practices ${ }^{15}$ and the use of global scores by some clinicians and for epidemiological purpose, this is a necessary component of such a synoptic report.

\section{Composite WHO grade/Gleason score}

The composite WHO grade / Gleason score is of targeted biopsies based on the combined grade of all cores taken from a single MRI focus or TRUS / DRE nodule, considering all cores as originating from a single tumour focus. ${ }^{16}$ In a similar manner, a composite score can also be applied in the systematic biopsy setting where $>1$ core is sampled from a single site and/or submitted in a single container. Again, the combined/composite WHO grade or GS can be used as opposed to individual GS on a per core basis. An example is as follows: three biopsies are taken from the right apex. Biopsy core 1 is GS $3+3$ in $80 \%$ of core, core 2 is GS $3+4$ in $40 \%$ of core, and core 3 is $4+4$ in $5 \%$ of core. Thus, a composite score for the three cores from the right apex would $3+4$.

\section{Adverse prognostic features}

Cribriform architecture carcinoma includes intraductal carcinoma and cribriform pattern 4. Both are recognized as adverse morphologic features in biopsies ${ }^{17-19}$ and their presence should be documented. The ability of MRI to identify these lesions is critical to ensure sampling of these aggressive sub-pathologies, thus for an individual target, their presence /absence should be recorded. Documentation of whether intraductal carcinoma has been included in grade assignment is also recommended as some pathologist's grade intraductal, and others do not. 9,20

\section{Tumour quantitation and extent of carcinoma}

Tumour quantitation needs to be reported in several ways for the following reasons: to support data inclusion in nomograms (eg the CAPRA score, MSKCC, EAU) and to determine active surveillance eligibility (some protocols define inclusion by the number of cores containing carcinoma). Firstly, the total number of cores sampled and the number of positive cores should be documented. Likewise, the number of positive sites and total number of sites sampled can be recorded. Thus for a positive Target biopsy, 5 of 5 cores may be involved but only $1 / 1$ site. For systematic cores, the usual method of core assessment would be employed (consider each site as separate) so that a standard 12 core biopsy with 1 core per site would still count as 12 cores and 12 sites.

The carcinoma extent within each core needs to be documented for systematic biopsies. This can be done as either millimetres of carcinoma or as percent core involvement. 
Additionally, the method by which the tumour has been measured needs to be documented eg considering multiple foci within the core as continuous (each focus is measured individually and the sum is regarded as the amount of core involvement) or discontinuous involvement (intervening benign stroma is included in the estimate of core involvement). This impacts the overall assessment of tumour volume. ${ }^{21,22}$

For an individual target, an aggregate measurement of carcinoma extent should be provided as either millimetres of tumor or as a percentage of tissue involved (see Fig 1). This is supported by a previous publication showing aggregate percentage assessment to be superior to individual core assessment ${ }^{8}$ in MRI target biopsies.

\section{Percent high grade (pattern 4, 5) carcinoma}

For ISUP / WHO grade 2 and 3 adenocarcinoma, the percentage of pattern 4 should be documented as low volume pattern $4(<10 \%)$ could qualify some patients as eligible for active surveillance. ${ }^{23}$ For all carcinomas $>$ WHO grade $1 /$ GS6, the percent high grade (GG4/5) component needs to be documented as it has prognostic impact ${ }^{24}$ and may be incorporated into algorithms (eg absolute percentage pattern 4) that predict for metastatic failure-free survival ${ }^{25}$

\section{Stage-related features}

The presence/absence of these features should be documented because of its unfavourable prognostic implications: perineural invasion, ${ }^{26}$ periprostatic fat involvement, ${ }^{27}$ seminal vesicle invasion and lymphovascular space invasion. ${ }^{28}$

\section{Target specific reporting}

The method of target detection should be specified eg MRI, ultrasound, digital rectal examination or other (eg PSMA-PET scan). For each target, a WHO grade/GS needs to be provided. We term these composite grades/GS to differentiate from the global grade assigned to the entire case (encompasses all targets $+/$ - systematic cores).

\section{Other features}

The presence of other histologic features can be noted in a separate "Additional features" category eg high grade prostatic intraepithelial neoplasia, atypical small acinar proliferation, adenosis and inflammation. For target biopsies, it is helpful to document the benign histologies present when the biopsy is negative for carcinoma as it has been demonstrated that increased stroma, chronic inflammation and atrophy can be associated with false positive findings on prostate MRI. ${ }^{29}$ 


\section{Conclusions}

The reporting of prostate biopsies is an evolving field and the advent of mpMRI and other technique driven target biopsies necessitates a change in practice. We propose a comprehensive synoptic report which can encompass both target and systematic biopsy cores to provide the optimal information for patient management. It is not our intention to advise on which elements any individual clinician should use in their management approach. We are advocating for comprehensive, complete synoptic reports that include all currently relevant data elements which will enable ANY clinician who uses ANY combination of biopsy data to access all the information they require in a single report. 


\section{References}

1. Siddiqui MM, Rais-Bahrami S, Truong H, et al. Magnetic resonance imaging/ultrasoundfusion biopsy significantly upgrades prostate cancer versus systematic 12-core transrectal ultrasound biopsy. Eur Urol 2013;64:713-19.

2. Kasivisvanathan V, Rannikko AS, Borghi M, et al. Mri-targeted or standard biopsy for prostate-cancer diagnosis. N Engl J Med 2018;378:1767-77.

3. Volkin D, Turkbey B, Hoang AN, et al. Multiparametric magnetic resonance imaging (mri) and subsequent mri/ultrasonography fusion-guided biopsy increase the detection of anteriorly located prostate cancers. BJU Int 2014;114:E43-E49.

4. Gordetsky JB, Nix JW, Rais-Bahrami S. Perineural invasion in prostate cancer is more frequently detected by multiparametric mri targeted biopsy compared with standard biopsy. Am J Surg Pathol 2016;40:490-94.

5. Zhao Y, Deng FM, Huang H, et al. Prostate cancers detected by magnetic resonance imaging-targeted biopsies have a higher percentage of gleason pattern 4 component and are less likely to be upgraded in radical prostatectomies. Arch Pathol Lab Med 2019;143:86-91.

6. Klotz L, Chin J, Black PC, et al. Comparison of multiparametric magnetic resonance imaging-targeted biopsy with systematic transrectal ultrasonography biopsy for biopsy-naive men at risk for prostate cancer: A phase 3 randomized clinical trial. JAMA Oncol 2021;7:534-42.

7. Klotz L, Pond G, Loblaw A, et al. Randomized study of systematic biopsy versus magnetic resonance imaging and targeted and systematic biopsy in men on active surveillance (asist): 2-year postbiopsy follow-up. Eur Urol 2020;77:311-17.

8. Gordetsky JB, Schultz L, Porter KK, et al. Defining the optimal method for reporting prostate cancer grade and tumor extent on magnetic resonance/ultrasound fusion-targeted biopsies. Hum Pathol 2018;76:68-75.

9. van Leenders G, van der Kwast TH, Grignon DJ, et al. The 2019 international society of urological pathology (isup) consensus conference on grading of prostatic carcinoma. Am J Surg Pathol 2020;44:e87-e99.

10. Prendeville S, Gertner M, Maganti M, et al. Role of magnetic resonance imaging targeted biopsy in detection of prostate cancer harboring adverse pathological features of intraductal carcinoma and invasive cribriform carcinoma. J Urol 2018;200:104-13.

11. Truong M, Feng C, Hollenberg G, et al. A comprehensive analysis of cribriform morphology on magnetic resonance imaging/ultrasound fusion biopsy correlated with radical prostatectomy specimens. J Urol 2018;199:106-13.

12. Athanazio D, Gotto G, Shea-Budgell M, et al. Global gleason grade groups in prostate cancer: Concordance of biopsy and radical prostatectomy grades and predictors of upgrade and downgrade. Histopathology 2017;70:1098-106.

13. Trpkov K, Sangkhamanon S, Yilmaz A, et al. Concordance of "case level" global, highest, and largest volume cancer grade group on needle biopsy versus grade group on radical prostatectomy. Am J Surg Pathol 2018;42:1522-29.

14. Varma M, Narahari K, Mason M, et al. Contemporary prostate biopsy reporting: Insights from a survey of clinicians' use of pathology data. J Clin Pathol 2018;71:874-78. 
15. Berney DM, Algaba F, Camparo $\mathrm{P}$, et al. The reasons behind variation in gleason grading of prostatic biopsies: Areas of agreement and misconception among 266 european pathologists. Histopathology 2014;64:405-11.

16. Arias-Stella JA, 3rd, Shah AB, Montoya-Cerrillo D, et al. Prostate biopsy and radical prostatectomy gleason score correlation in heterogenous tumors: Proposal for a composite gleason score. Am J Surg Pathol 2015;39:1213-18.

17. Downes MR, Xu B, van der Kwast TH. Cribriform architecture prostatic adenocarcinoma in needle biopsies is a strong independent predictor for lymph node metastases in radical prostatectomy. Eur J Cancer 2021;148:432-39.

18. Watts K, Li J, Magi-Galluzzi C, et al. Incidence and clinicopathological characteristics of intraductal carcinoma detected in prostate biopsies: A prospective cohort study. Histopathology 2013;63:574-79.

19. Van der Kwast T, Al Daoud N, Collette L, et al. Biopsy diagnosis of intraductal carcinoma is prognostic in intermediate and high risk prostate cancer patients treated by radiotherapy. Eur J Cancer 2012;48:1318-25.

20. Epstein JI, Amin MB, Fine SW, et al. The 2019 genitourinary pathology society (gups) white paper on contemporary grading of prostate cancer. Arch Pathol Lab Med 2021;145:461-93.

21. Brimo F, Vollmer RT, Corcos J, et al. Prognostic value of various morphometric measurements of tumour extent in prostate needle core tissue. Histopathology 2008;53:17783.

22. Karram S, Trock BJ, Netto GJ, et al. Should intervening benign tissue be included in the measurement of discontinuous foci of cancer on prostate needle biopsy? Correlation with radical prostatectomy findings. Am J Surg Pathol 2011;35:1351-55.

23. Morash C, Tey R, Agbassi C, et al. Active surveillance for the management of localized prostate cancer: Guideline recommendations. Can Urol Assoc J 2015;9:171-78.

24. Sauter G, Steurer S, Clauditz TS, et al. Clinical utility of quantitative gleason grading in prostate biopsies and prostatectomy specimens. Eur Urol 2016;69:592-98.

25. Martell K, Mendez LC, Chung H, et al. Absolute percentage of biopsied tissue positive for gleason pattern 4 disease (app4) appears predictive of disease control after high dose rate brachytherapy and external beam radiotherapy in intermediate risk prostate cancer. Radiother Oncol 2019;135:170-77.

26. Delahunt B, Murray JD, Steigler A, et al. Perineural invasion by prostate adenocarcinoma in needle biopsies predicts bone metastasis: Ten year data from the trog 03.04 radar trial. Histopathology 2020;77:284-92.

27. Goldberg H, Ramiz AH, Glicksman R, et al. Extraprostatic extension in core biopsies epitomizes high-risk but locally treatable prostate cancer. Eur Urol Oncol 2019;2:88-96.

28. Saeter T, Vlatkovic L, Waaler G, et al. Combining lymphovascular invasion with reactive stromal grade predicts prostate cancer mortality. Prostate 2016;76:1088-94.

29. Gordetsky JB, Ullman D, Schultz L, et al. Histologic findings associated with false-positive multiparametric magnetic resonance imaging performed for prostate cancer detection. Hum Pathol 2019;83:159-65. 


\section{Figures and Tables}

Fig. 1. Diagrammatic representation of composite calculation of Gleason score/WHO grade and tumour volume in a target biopsy.

\section{Calculation of Composite Extent in a Targeted Biopsy}

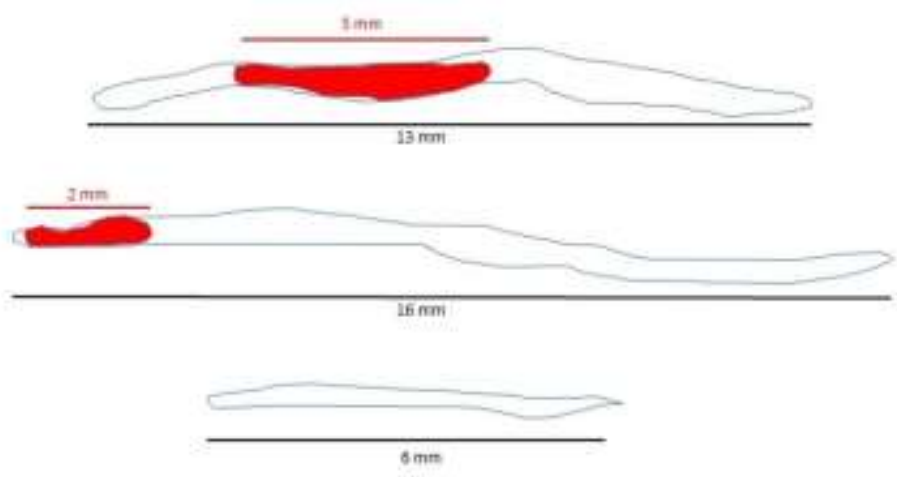

Target site with $2 / 3$ cores positive for carcinoma Composite extent in mm: $5+2=7 \mathrm{~mm}$

Composite percentage is $(5+2) /(13+16+6)=20 \%$ 